\title{
Zika-related knowledge, attitudes, and practices: Programmatic implications for Zika prevention in El Salvador
}

Breakthrough RESEARCH

Follow this and additional works at: https://knowledgecommons.popcouncil.org/departments_sbsr-pgy How does access to this work benefit you? Let us know!

\section{Recommended Citation}

Breakthrough RESEARCH. 2019. "Zika-related knowledge, attitudes, and practices: Programmatic implications for Zika prevention in El Salvador," Programmatic Research Brief. Washington, DC: Population Council. 


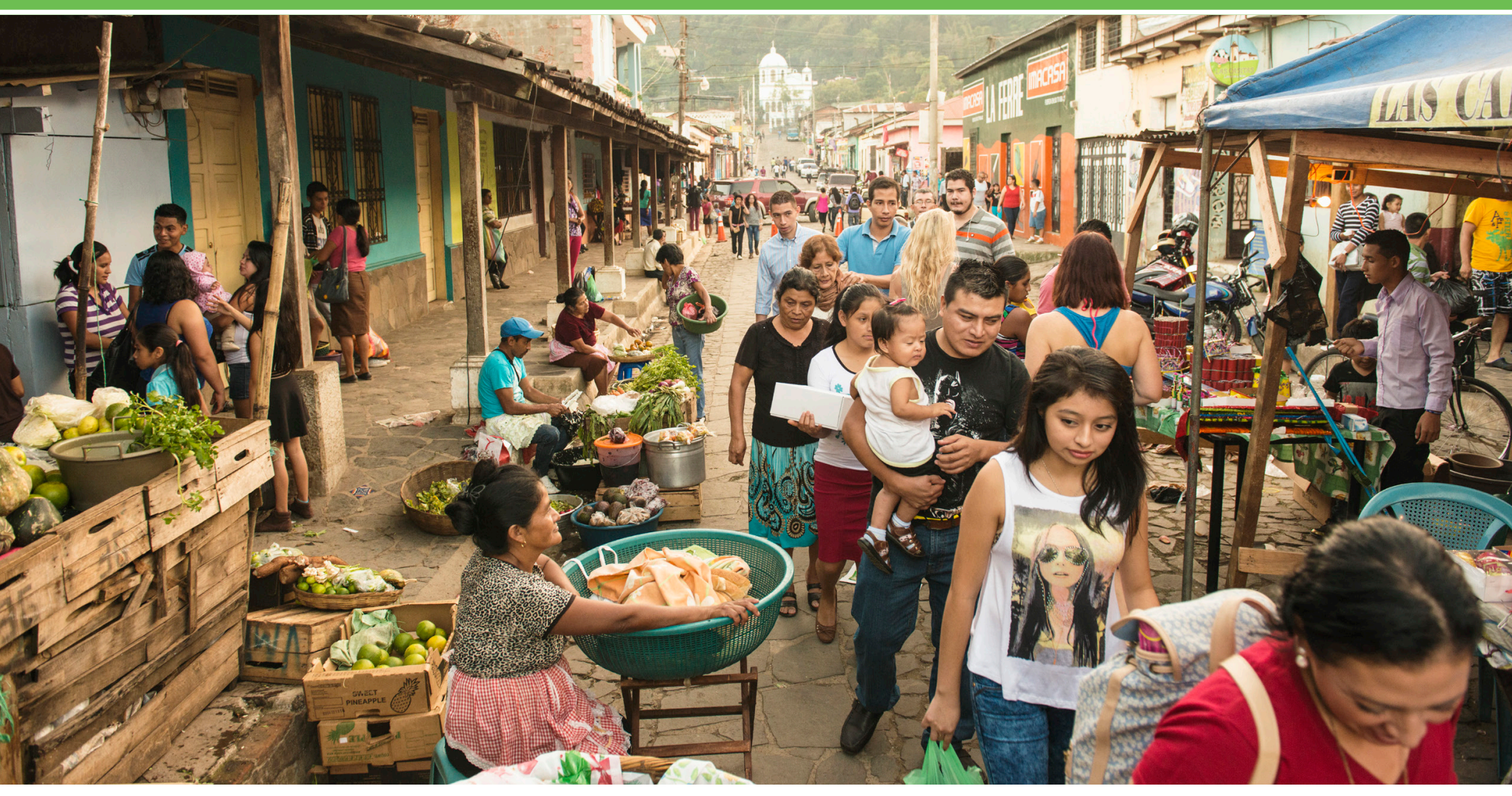

\section{Zika-Related Knowledge,} Attitudes, and Practices

This brief provides key insights for implementers of social and behavior change (SBC) activities for Zika prevention in El Salvador. The insights are based on data from a representative sample of people living in United States Agency for International Development (USAID) Zika program implementation communities and support an evidence-based approach for programming directed toward increasing the practice of prevention behaviors for Zika and other Aedes aegypti-transmitted viruses. The data indicate a need to: (1) narrow existing knowledge gaps related to the Zika virus, particularly its health consequences and effective personal preventive practices; (2) communicate more specifically about preventive behaviors to enable effective action; and (3) leverage social norms surrounding prevention of Zika to promote individual action. 


\section{Background}

The first outbreak of Zika detected in the Americas occurred in 2015, with a spike in suspected congenital malformations and other neurological complications. ${ }^{1}$ In April 2016, the U.S. Department of State dedicated funding for the USAID Zika response, prioritizing prevention efforts and programming to minimize negative pregnancy outcomes in affected countries.

A key component of the USAID Zika Response is SBC programming that aims to promote the uptake of effective prevention behaviors to reduce Zika transmission. Programs emphasize ensuring personal protection for pregnant women, engaging high-risk communities in prevention practices, and improving women's demand for and access to antenatal care services. ${ }^{*}$

\section{Why Did We Collect Information on Knowledge, Attitudes, and Practices?}

Behavior change theories help us understand what enables and motivates people to act the way they do. ${ }^{4}$ The social-ecological model of behavior change highlights the importance of individual factors (such as knowledge, perceptions, and attitudes about a disease and how to prevent it), community-level factors (such as normative environment), and social and structural factors (such as access to resources and services) for understanding whether or not a person carries out a particular behavior. The extended parallel processing model highlights how a person's perception of risk associated with a disease, as well as their beliefs in the effectiveness of solutions and their own confidence to practice them, will influence whether people will take preventive action. ${ }^{4}$ These and other theories provide the groundwork to measure constructs that help SBC implementers make decisions about how best to influence behavior within target communities.

\section{How Was the Data Collected?}

In 2018, the Breakthrough RESEARCH project conducted cross-sectional household surveys in Guatemala, Honduras, El Salvador, and the Dominican Republic. The survey assessed knowledge, attitudes, and practices among other factors related to Zika and other diseases transmitted by the Aedes aegypti mosquito. The multistage cluster random sample included men and women ages 18 to 49 living in USAID program implementation areas. In El Salvador, the survey

\footnotetext{
* For more information visit www.usaid.gov/what-we-do/global-health/zika.
}

\section{WHY ZIKA MATTERS}

- Zika virus (ZIKV) is a communicable disease spread by Aedes aegypti mosquitoes, which also transmit other arboviruses including dengue and chikungunya.

- ZIKV can also be transmitted through sexual intercourse and from pregnant mother to unborn child. ${ }^{1}$ ZIKV is known to cause neurological impacts, such as Congenital Zika Syndrome (CZS).

- Many people infected with ZIKV do not develop symptoms, leading to a lower perceived risk of infection. ${ }^{2}$

was carried out between August and October 2018 within implementation areas of six provinces: Chalatenango, San Miguel, Ahuachapan, Sonsonate, Cabañas, and La Libertad. Interviews were conducted with 672 individuals, of which 32 percent were men and 68 percent women. Data were weighted by sociodemographic characteristics to reflect the population in the implementation areas.

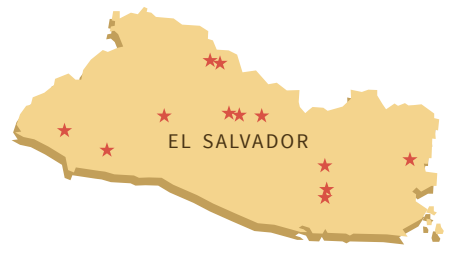

This study collected information in five main areas:

- Sociodemographics: Age, education, sex, household assets.

- Knowledge: Transmission, health effects, prevention for each disease.

- Attitudes: Perceptions of disease risk, effectiveness of prevention behaviors, feasibility of conducting prevention behaviors.

- Self-reported prevention practices: Behaviors to prevent mosquito bites and mosquito breeding in the last 30 days and last seven days.

- Observed practices: Observation of the use of secure lids for water storage containers in which mosquitoes are known to breed. 


\section{Programmatic Implications}

These insights for SBC programs are based on key findings from the surveys in El Salvador.

1. Prevention programming and messages should emphasize the links between the Zika virus and birth defects, including CZS, especially among the male population, as it is a key differentiating factor between Zika and other arboviruses. For pregnant couples, having a healthy child may be a key motivator for prevention.

2. Prevention programming should prioritize behaviors best known to be effective against Zika, particularly behaviors for personal protection during pregnancy. Messages should contain specific instructions for complex preventive behaviors.

3. Prevention programming should continue to highlight Zika's ability to be sexually transmitted, particularly among pregnant women and their partners, emphasizing the importance of condom use for preventing transmission during pregnancy.

4. Prevention programming should specifically target men, particularly given men's role in the negotiation of condom use for prevention.

5. Prevention programming should include messages that continue to specify that mosquitoes can bite at any time to emphasize continuous use of personal protection such as repellents, especially for pregnant women and their partners.

6. Prevention programming should highlight and design for ways in which behaviors can be made easier, enhancing both their perceived and actual feasibility for adoption.

7. Prevention programming should continue to promote Zika prevention as a pillar of community identity, leveraging the effects of descriptive social norms on people's behavior.

\section{Key Findings on Zika Knowledge}

\section{Awareness of Disease}

The majority of people surveyed had heard about Zika, dengue, or chikungunya, and 83 percent of people had heard about all three diseases. However, out of the three, Zika was the least commonly known (see Table 1).

\begin{tabular}{|c|c|}
\hline DISEASE & $\begin{array}{c}\text { \% OF PEOPLE WHO HAD EVER } \\
\text { HEARD OF DISEASE }\end{array}$ \\
\hline Zika & $88 \%$ \\
\hline Dengue & $95 \%$ \\
\hline Chikungunya & $92 \%$ \\
\hline
\end{tabular}

\section{Transmission and Health Effects}

By mosquitoes: Among those who had heard of each disease, nearly everyone knew that mosquitoes transmit Zika (96 percent), dengue (100 percent), and chikungunya (97 percent). Over half (56 percent) of those aware that Zika is transmitted via a mosquito bite knew that the mosquito can bite at any time of the day or night. Women were less likely to know mosquitoes that transmit Zika can bite at any time of day or night compared to men (48 percent v. 64 percent, $\mathrm{p}<.05)^{+}$

Through sexual and vertical transmission: Only 15 percent of participants who had heard about Zika knew that it could be sexually transmitted, and 6 percent knew that it could be transmitted from mother to child during pregnancy.

Only 14 percent of people who were aware of Zika identified birth defects as a potential adverse health outcome. Men were significantly less aware that birth defects can be a health effect of Zika compared to women (10 percent v. 18 percent, $p<.05)$.

\section{Prevention Methods}

The most commonly listed behaviors with a high potential to prevent Zika were clearing stagnant water (87 percent), cleaning water storage containers (74 percent), changing

+ Chi-square test for independence was used for all bivariate analyses. 
standing water in storage containers (60 percent), covering water storage containers (49 percent), and cleaning trash that harbors mosquitoes (deschatarrización) outside of the home (43 percent). ${ }^{\ddagger}$

Only 28 percent of participants identified repellent and less than 2 percent identified condoms as a Zika prevention mechanism.

Significantly more men than women were able to identify cleaning containers (78 percent v. 71 percent, $p<.001$ ) as a preventive behavior, but fewer men than women identified using condoms as a preventive behavior (.03 percent v. 3.0 percent, $p<.001$ ). Compared to the wealthiest people surveyed, fewer of the most economically vulnerable individuals (those in the poorest quintile) were able to identify repellent use as a preventive behavior (16 percent v. 47 percent, $p<.01$ ). The same is true for changing water (26 percent v. 71 percent, $p<.001$ ), cleaning containers (46 percent v. 74 percent, $p<.05$ ), and covering containers (28 percent v. 65 percent, $p<.05)$.

\section{Key Findings on Attitudes About Zika}

\section{Perceived Risk of Zika, Dengue, and Chikungunya}

The overwhelming majority of people surveyed perceived themselves to be at risk of Zika to some degree, whether it was low, medium, or high. Very few people reported no risk or did not know their risk of Zika, and even fewer reported the same for dengue and chikungunya. Approximately onethird of participants perceive themselves to be at high risk

\section{FIGURE 1. RISK PERCEPTION BY DISEASE}

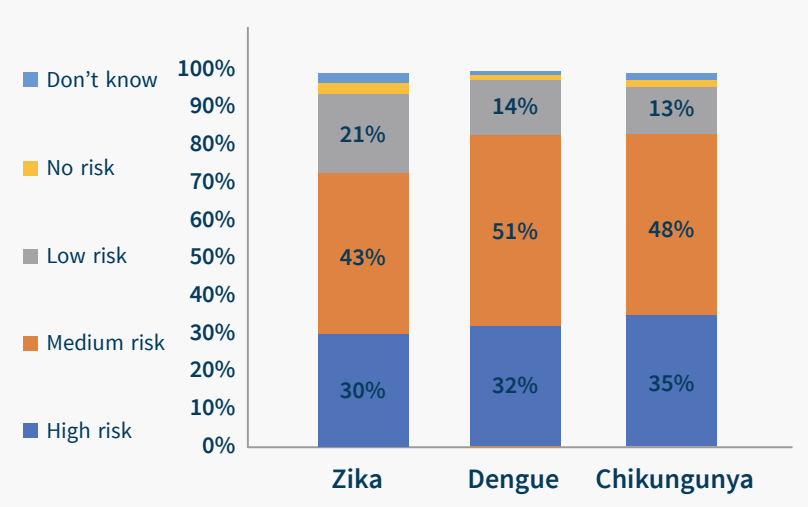

‡ Effective behaviors: repellent use, clearing stagnant water, cleaning water storage containers, covering water storage containers, using larvicide, and using screens on windows and doors. of Zika (30 percent), dengue (32 percent), or chikungunya (35 percent), as presented in Figure 1. Slightly less than half of participants reported to be at medium risk of Zika (43 percent) and chikungunya (48 percent), and more than half reported a medium level of risk for dengue (51 percent).

In addition, most participants reported that they would be very concerned if they contracted Zika (80 percent), dengue (80 percent), and chikungunya (81 percent).

\section{Perceived Feasibility of Prevention Behaviors}

When asked about their perceptions of how easy it is to carry out certain prevention behaviors listed in Table 2, the majority of respondents do not report behaviors as being very easy. Less than 8 percent of people in this study perceived it was easy to use condoms in general or during pregnancy, or to use repellents or larvicide.

TABLE 2. PERCENTAGE OF PEOPLE REPORTING PREVENTIVE BEHAVIOR TO BE VERY EASY

\begin{tabular}{l|c|}
\hline BEHAVIOR & $\%$ \\
\hline Change water & $25 \%$ \\
\hline Clean water containers & $22 \%$ \\
\hline Cover water containers & $22 \%$ \\
\hline Remove stagnant water in the community & $17 \%$ \\
\hline Remove stagnant water at home & $16 \%$ \\
\hline Use condoms in general & $8 \%$ \\
\hline Use repellent & $8 \%$ \\
\hline Use condoms during pregnancy & $7 \%$ \\
\hline Use larvicide & $7 \%$ \\
\hline
\end{tabular}

\section{Perceived Effectiveness of Prevention Behaviors}

When asked to list known prevention behaviors by how effective they are to prevent mosquito breeding and illnesses such as Zika, three-quarters of participants thought clearing stagnant water was the most effective prevention method, followed by cleaning water storage containers (43 percent) and changing purposefully kept stagnant water (32 percent) (see Table 3). 
TABLE 3. PERCENTAGE OF PEOPLE WHO PERCEIVE BEHAVIOR AS AMONG THE TOP THREE MOST EFFECTIVE

\begin{tabular}{|l|c|}
\hline BEHAVIOR & $\%$ \\
\hline Clear stagnant water & $75 \%$ \\
\hline Clean water storage containers & $43 \%$ \\
\hline Change stagnant water & $32 \%$ \\
\hline Use bed nets & $27 \%$ \\
\hline Clear trash outside the house & $25 \%$ \\
\hline Use repellent & $23 \%$ \\
\hline Use mosquito spray (e.g. Raid ${ }^{\circledR}$ ) & $17 \%$ \\
\hline Cover water containers & $10 \%$ \\
\hline Use larvicide & $9 \%$ \\
\hline Use screens on windows and doors & $6 \%$ \\
\hline Use condoms & $5 \%$ \\
\hline Use coils & $4 \%$ \\
\hline
\end{tabular}

Female respondents were significantly more likely to perceive cleaning water storage containers as among the top three most effective behaviors than men $(p<.05)$.

\section{Key Findings on Prevention Practices}

\section{Practices to Prevent the Sexual Transmission of Zika}

Although slightly more than one-fifth of people surveyed (22 percent) reported using condoms during sex in the last 30 days, only 2 percent of people surveyed reported using condoms to prevent Zika.

\section{Practices to Prevent Mosquito Bites and Mosquito Breeding}

Out of people interviewed:

- 72 percent of all participants had done at least one behavior that had a high potential to be effective against mosquitoes in the last $\mathbf{3 0}$ days.
- 75 percent of all participants carried out at least one behavior that had a high potential to be effective against mosquitoes in the last seven days.

The most common responses to an open-ended question about prevention behaviors carried out in the last 30 days and last seven days are presented in Figure 2. The most commonly reported behavior is eliminating stagnant water, while the least common is using larvicide in water storage containers. Behaviors with the greatest potential to be effective are marked with an asterisk.

Women were 9 percentage points more likely to have cleaned their water storage container than men in the last 30 days $(p=.019)$. This finding confirms gender normative roles, where women are mostly in charge of household chores.

\section{FIGURE 2. PREVENTION BEHAVIORS IN LAST 30 AND LAST SEVEN DAYS}

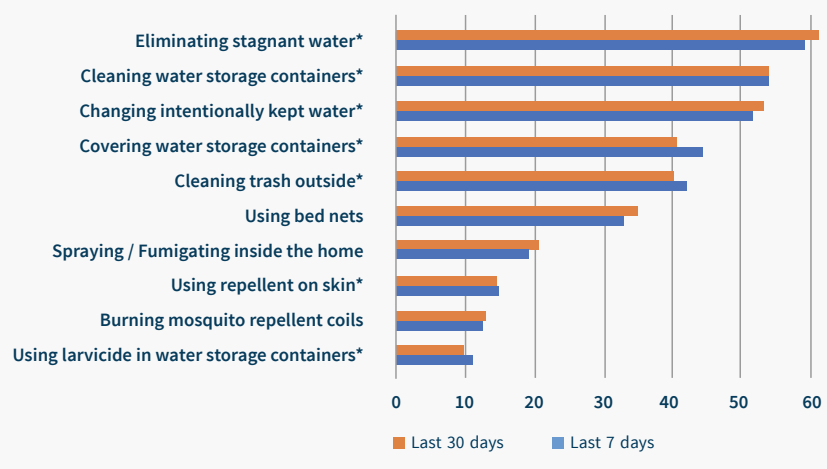

About one in five people surveyed (21 percent) reported not carrying out any type of mosquito prevention behavior in the past 30 days. Among them, the most common explanation given was not having enough time or being too busy (43 percent). For repellent use specifically, the most salient barriers were cost and access to repellent, with 45 percent of those who had not used repellent in the last seven days saying they did not have the materials and 29 percent saying the cost of repellent was too high to use it.

\section{Practices Concerning Water Storage Containers}

To understand how people in El Salvador clean their water storage containers, we asked those who reported doing so in the last seven days to specify steps taken. Table 4 shows in rank order the actions reported to clean water storage containers. Over half of respondents (62 percent) mentioned emptying the container as a step to cleaning it, scrubbing the walls of the container with a brush (57 percent), rinsing the 
container (56 percent), and applying bleach to the walls of the container ( 55 percent) as well as detergent ( 55 percent).

\begin{tabular}{|c|c|}
\hline ACTIONS & $\%$ \\
\hline Empty the container & $62 \%$ \\
\hline Scrub walls with brush & $57 \%$ \\
\hline Rinse container & $56 \%$ \\
\hline Apply bleach to walls of container & $55 \%$ \\
\hline Apply detergent to walls of container & $55 \%$ \\
\hline Add bleach to the water & $25 \%$ \\
\hline Let ingredients act for a few minutes & $23 \%$ \\
\hline
\end{tabular}

Twenty-five percent of people added bleach to water, which is not an effective means of eliminating mosquito larvae or pupae, and only 23 percent of respondents reported letting cleaning ingredients act on the surface of the water storage container for a few minutes.

In addition to the survey, data were collected through observation of storage containers in participants' homes. Out of 672 respondents, 582 (87 percent) allowed surveyors to view their water storage containers. These included 662 wash basins (pilas) and 332 long-term water storage containers (commonly plastic drums). Surveyors observed containers for covers to assess the quality of the lids used. For a container to be effectively covered, lids must cover the container completely and fit tightly to avoid any gaps at any point in the circumference of the lid. If they are made from hard plastic, they must not be warped or allow water to pool on their surface. If they are made from a malleable material, they must not touch the surface of the water to form secondary breeding sites.

Overall, 8 percent of observed wash basins and 11 percent of water storage containers were found to have mosquito larvae. Wash basins that were effectively covered were significantly less likely to have mosquito larvae at the time of observation than wash basins that were not effectively covered (were not covered or had a cover that did not meet the effectiveness criteria). Only 0.5 percent of effectively covered wash basins had larvae, compared to 9 percent of wash basins without an effective cover $(p<.001)$ (see Table 5 ).
Similarly, long-term storage containers that were effectively covered were significantly less likely to have mosquito larvae at the time of observation than those not effectively covered.

Two percent of effectively covered containers had larvae, compared to 26 percent of containers that were not effectively covered $(p<.001)$. This effect holds true even when controlling for self-reported cleaning of containers in the last 30 days.

\begin{tabular}{|c|c|}
\hline WASH BASINS & $\%$ \\
\hline Effectively Covered & $0.5 \%$ \\
\hline Not Effectively Covered & $9 \%$ \\
\hline \multicolumn{2}{|c|}{ LONG-TERM STORAGE CONTAINERS } \\
\hline Effectively Covered & $2 \%$ \\
\hline Not Effectively Covered & $26 \%$ \\
\hline
\end{tabular}

\section{Associations with Prevention Behaviors ${ }^{\S}$}

In El Salvador the most consistent factor associated with acting to prevent mosquito biting or breeding is knowledge of effective prevention behaviors. Using a scale measuring the number of Zika prevention behaviors known, we found that people who knew of eight different prevention behaviors were 35 percentage points more likely to engage in preventive behaviors than those who did not know any. Increased knowledge of effective prevention behaviors is significantly associated with greater self-reported use of covering water storage containers $(p<.001)$, clearing stagnant water $(p<.001)$, cleaning water storage containers $(p<.001)$, and repellent use $(p<.01)$. However, the strength of the association between knowledge of prevention behaviors and carrying them out varied by the type of behavior. In the case of repellent use, approximately two-thirds of people with high knowledge of prevention behaviors did not use repellents (65 percent), despite their being a highly preventive measure. Therefore, knowledge alone is insufficient to ensure behavior adoption across all behaviors.

When looking at people who had carried out any effective behavior in the last seven days regardless of which one it

$\S$ Results in this section were derived from logistic regression analyses. 
was, descriptive social norms (i.e., perceptions about what members of your community are doing) were found to be relevant. Controlling for age, sex, education, wealth, knowledge of Zika, exposure to home visits by prevention technician or volunteer, and exposure to media, respondents who strongly agreed that most people in their community did something to prevent Zika were 13 percentage points more likely to have carried out an effective preventive behavior in the last seven days ( $p<.001)$. Exploring the individual relationship between descriptive social norms and preventive behaviors, we see significant associations particularly with clearing stagnant water and cleaning water storage containers. Compared to people who strongly agreed that most people in their community did something to prevent Zika, those who disagreed were 26 percentage points less likely to have cleaned their water storage containers ( $p=.005)$, and 22 percentage points less likely to have cleared stagnant water in the last thirty days $(p=.01)$.

Despite strong bivariate associations between receiving a home visit for mosquito prevention by volunteers or technicians and carrying out preventive actions, when explored through multivariate analysis, preventive behaviors' perceived effectiveness and feasibility alone are significantly associated with action. People who believed that repellent use, cleaning water storage containers, or clearing stagnant water were among the most effective preventive measures were 15 percentage points, 14 percentage points, and 8 percentage points, respectively, more likely to have used repellents $(p<.001, p<.001, p=.04)$. Likewise, controlling for levels of wealth, those who believed using repellents was very easy were 7 percentage points more likely to use it $(p=.04)$, and those who believed clearing stagnant water was very easy were 14 percentage points more likely to do it $(p<.001)$.

\section{Implications for Action}

Within the surveyed areas in El Salvador, a large majority of people have heard about Zika, and almost all who have heard of the virus know that it can be transmitted by a mosquito. Despite this high level of general awareness, only about half of the people surveyed knew that mosquitoes can bite at any time. Personal prevention messages promoting the use of repellents should continue to specify the need to protect against mosquito bites at any time of day, especially among pregnant women. ${ }^{7}$ Additionally, knowledge of sexual transmission is very low, and few people reportedly know of the link between Zika disease during pregnancy and adverse pregnancy outcomes. Even though sexual transmission has been estimated to account for only 4 percent to 5 percent of overall Zika transmission, the implications of transmission during pregnancy are so severe that programs must continue to raise awareness of both sexual transmission of Zika and its potential health effects during pregnancy, particularly among pregnant women and their partners. ${ }^{5,6}$ Men must be specifically targeted with messages explaining the potential health effects of Zika during pregnancy, given their role in the negotiation of condom use for prevention.

Knowledge of most effective vector control behaviors is high, yet knowledge of personal protective behaviors, such as repellent and condom use, is low. People are practically unaware of condom use as a mechanism to protect against Zika. Perceived feasibility-an important construct in promoting behavior change-is also especially low for personal protection measures that are most effective. ${ }^{7}$ Prevention programming should highlight and design ways in which behaviors can be made easier to do, enhancing both their perceived and actual feasibility for adoption.

A large majority of the people surveyed have carried out at least one effective preventive behavior. Yet, as was seen with knowledge of personal protective behaviors, the practice of these behaviors lags behind vector control behaviors. Programs should continue prioritizing activities aimed at raising awareness of specific behaviors to prevent Zika, particularly those personal protective behaviors that are especially important during pregnancy. Conversely, even when general knowledge and practice of vector control behaviors are high, messages should contain specific instructions for practicing complex preventive behaviors. ${ }^{7,8}$ Missed steps in vector control behaviors, like scrubbing water storage containers and effectively covering containers, are crucial for effectively eliminating mosquito breeding sites.

Lastly, the importance of community social norms, specifically what people believe others in their community are doing, is an important element in SBC theory and confirmed in El Salvador. People who agreed that others in their community did something to prevent Zika had a higher likelihood of enacting effective prevention behaviors. Prevention messages should continue to promote Zika prevention as a pillar of community identity, leveraging the effects of descriptive social norms on people's behavior. 


\section{References}

1 World Health Organization (WHO). 2016. Zika Strategic Response Plan. Geneva, Switzerland: WHO.

2 Haby, Michelle M. et al. 2018. "Prevalence of asymptomatic Zika virus infection: a systematic review," Bulletin of the World Health Organization 96: 402-413D.

3 Centers for Disease Control and Prevention (CDC). 2018. Congenital Zika Syndrome \& Other Birth Defects. Atlanta: CDC. Available from www.cdc.gov/pregnancy/ zika/testing-follow-up/zika-syndrome-birth-defects. html.

4 Health Communication Capacity Collaborative. 2016. Social and Behavior Change Communication for Emergency Preparedness Implementation Kit. Baltimore: Johns Hopkins University (JHU). Available from: http://healthcommcapacity.org/wp-content/ uploads/2017/01/SBCCforEP I-Kit.pdf.

5 Coelho, Flavio Codeco et al. 2016. "Higher incidence of Zika in adult women than adult men in Rio de Janeiro suggests a significant contribution of sexual transmission from men to women," International Journal of Infectious Diseases 51: 128-132.

6 Rao, Rashmi et al. 2017. "Zika risk and pregnancy in clinical practice: ongoing experience as the outbreak evolves," Obstetrics and Gynecology 129(6): 10981103.

7 Breakthrough ACTION+RESEARCH. 2018. Zika Prevention Behavior Matrix. Baltimore: JHU. Available from www.zikacommunicationnetwork.org/resources/ zika-prevention-behavior-matrix.

8 Breakthrough ACTION+RESEARCH. 2018. Technical Specification Content Guide for Behaviors With a High Potential to Prevent Zika. Baltimore: JHU. Available from www.zikacommunicationnetwork.org/resources/technical-specifications-content-guide-behaviors-high-potential-prevent-zika.

\section{Acknowledgements}

This programmatic research brief describes work led by Tulane University under Breakthrough RESEARCH in collaboration with TEPHINET in El Salvador. The brief was edited and designed by Population Reference Bureau (PRB).

\section{Suggested citation:}

Breakthrough RESEARCH. 2019. "Zika-Related Knowledge, Attitudes, and Practices: Programmatic Implications for Zika Prevention in El Salvador," Programmatic Research Brief. Washington, DC: Population Council.

Photo credits:

helovi, Getty Images

(C) 2019 The Population Council. All rights reserved

Breakthrough RESEARCH, Population Council

4301 Connecticut Ave., NW, Suite 280 | Washington, DC 20008 +12022379400 | breakthroughactionandresearch.org

BreakthroughRESEARCH@popcouncil.org

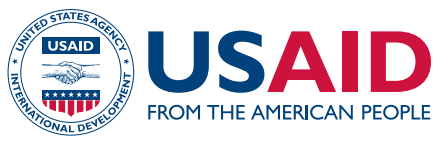

Breakthrough RESEARCH is made possible by the generous support of the American people through the United States Agency for International Development (USAID) under the terms of cooperative agreement no. AIDOAA-A-17-00018. The contents of this document are the sole responsibility of the Breakthrough RESEARCH and Population Council and do not necessarily reflect the views of USAID or the United States Government.

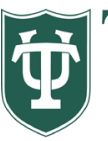

Tulane University

P

POPULATION COUNCIL

Ideas. Evidence. Impact.

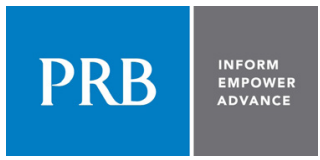

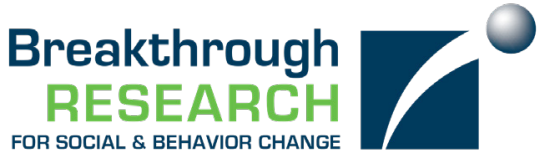

Breakthrough RESEARCH catalyzes social and behavior change (SBC) by conducting state-ofthe-art research and evaluation and promoting evidence-based solutions to improve health and development programs around the world. Breakthrough RESEARCH is a consortium led by the Population Council in partnership with Avenir Health, ideas42, Institute for Reproductive Health at Georgetown University, Population Reference Bureau, and Tulane University. 